\title{
IMPLEMENTATION OF A DOUBLE AC/DC/AC CONVERTER WITH POWER FACTOR CORRECTION (PFC) FOR NON-LINEAR LOAD APPLICATIONS
}

\author{
E.Alvear ${ }^{1}$, M.Sanchez ${ }^{1}$ and J.Posada ${ }^{2}$ \\ ${ }^{1}$ Department of Automation and Electronics, \\ Electronics Engineering, Universidad Autónoma de Occidente \\ Campus Valle del Lili - Cali, Km. 2 Vía Cali- Jamundi (Colombia) \\ Phone/Fax number: +57 2 3188000, e-mail: edwinalvear@yahoo.com.mx,msanchez_237@yahoo.es \\ ${ }^{2}$ Director of the electrical machines and power electronics area of the group of investigation in energies GIEN \\ Universidad Autónoma de Occidente \\ Campus Valle del Lili - Cali, Km. 2 Vía Cali- Jamundi (Colombia) \\ Phone: +5723188000 Ext.11853, e-mail: jposada@uao.edu.co
}

\begin{abstract}
The double conversion circuits are widely used as motors drivers, UPS's, Active filters, etc. As its name indicates, there are two types of conversions in this system, the $\mathrm{AC} / \mathrm{DC}$ and the $\mathrm{DC} / \mathrm{AC}$ conversion. In this paper the results obtained of the implementation of a double conversion system $\mathrm{AC} / \mathrm{DC} / \mathrm{AC}$ are presented. For the AC/DC conversion a topology with power factor correction (PFC) is used, which is controlled by the new technique OCC (One Cycle Control). The previously mention technique showed similar results in comparison with the traditional control technique "The Multiplier based approach", but with the advantages of less complexity and reduction in component count.
\end{abstract}

In the $\mathrm{DC} / \mathrm{AC}$ conversion an inverter circuit with the modulation technique SPWM was implemented, in which we obtained a regulated output $\mathrm{AC}$ voltage and a low harmonic content.

\section{Key words}

AC/DC/AC conversion, PFC, SPWM, THD, harmonics.

\section{Introduction}

Nowadays, the electrical energy demand is increasing so fast, that it threatens even to exceed the installed capacity of the national electrical system generating plants. Another equally important issue is that due to the staggering development of the electronics industry, most of the AC grid connections are from electronics equipments. Such equipments use traditional power supply (static rectifiers), which demands a harmonic-rich AC-line current. These equipments run with a low power factor (PF) and a high total harmonic distortion (THD). Working with a low power factor means that the equipment does not make the most of the supplied energy.
On the other hand, high harmonic distortion contaminates the AC grid, and it can cause electrical failures by interference, in susceptible equipment; in other words, the electrical energy is not being used properly. The previously mentioned negative effects of line current distortion have prompted a need for setting limits for the line current harmonics of equipment connected to the electricity distribution network. Thus the need to develop double AC/DC/AC conversions systems with power factor correction.

There are two types of conversions systems, the AC/DC conversion system and the $\mathrm{DC} / \mathrm{AC}$ conversion system. The AC/DC conversion system takes the alternating current (AC) to convert it into direct current (DC); this conversion can be done with static rectifiers systems, which produces a non-sinusoidal line current due to the non-linear input characteristic leading to a low power factor; or with power factor correction systems (PFC). In the DC/AC conversion it is used an inverter circuit, which uses a modulation technique for its control, wherein parameters like conversion efficiency, harmonic content and switching losses, are relevant points in the design.

In this paper, for the $\mathrm{AC} / \mathrm{DC}$ conversion a power factor corrector system is implemented, which has a continuous voltage, with its amplitude greater than the peak input signal and which avoids the injection of current harmonic to the AC grid.

\section{AC/DC Conversion}

The AC-DC rectification consisting of a diode bridge followed by a filter capacitor is cheap and robust, but demands a harmonic-rich ac-line current, as it is shown in figure 1 .

One way to solve this problem is to use converters with active power factor (APFC). An APFC basically is a 
SMPS controlled to draw an input current with little loworder harmonics, which consist on a diode bridge, followed by a $\mathrm{DC} / \mathrm{DC}$ converter with an appropriate control.

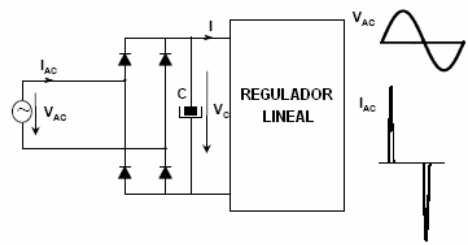

Fig. 1. Power supply of a typical electronics load.[1]

If the controller forces the input current to have the same shape as the input voltage so that the input impedance appears to be resistive, that converter is called a resistor emulator (RE). Among all basic topologies which has RE characteristics, the most popular is the Boost converter, due to its simplicity and robustness, as it is shown in figure 2 .

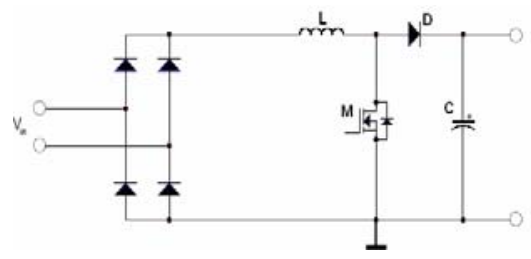

Fig. 2. Boost converter topology. [2]

The voltage gain of this converter is given by:

$$
V_{o}=\frac{V_{s}}{1-D}
$$

Where $V_{O}$ is the output voltage, $V_{S}$ is the input $\mathrm{AC}$ voltage and $D$ is the duty cycle, which is in the range from 0 to 1 .

There are two traditional approaches to control a resistor emulator, namely, the voltage follower approach and the multiplier approach. The voltage-follower approach realizes a resistor emulator with the constant-duty-ratio or the constant-ON-time control, such as a flyback or a Cuk converter operating at discontinuous-conduction mode (DCM), or a Boost converter at the boundary of DCM and continuous-conduction mode (CCM). The control circuit is simply a voltage mode pulse widthmodulation (PWM) chip which does not even require a current sensor. However, the DCM or the boundary operation causes a large current stress on semiconductors and demands more effort to attenuate the current ripple so as to have a satisfactory low electromagnetic interference (EMI) to the line. The input-current ripple of the DCM Cuk converter introduced in can be eliminated by inductor coupling; however, the current stress on semiconductors remains the same. At high-power applications, the current stress and current ripple become too large for a single DCM converter to operate efficiently. Therefore, the voltage-follower approach is not suitable for high-power application. On the other hand, typical PFC implementation for medium to high power systems is done using the multiplier based approach, which relatively requires complicated control circuitry [3].

The One Cycle Control is a new technique that has shown the same results in performance and efficiency, comparing with the multiplier based approach, but the One Cycle Control has the advantages of less complexity and reduction in component count. The basic schematic diagram of this control technique for a Boost converter is shown in figure 3 [4].

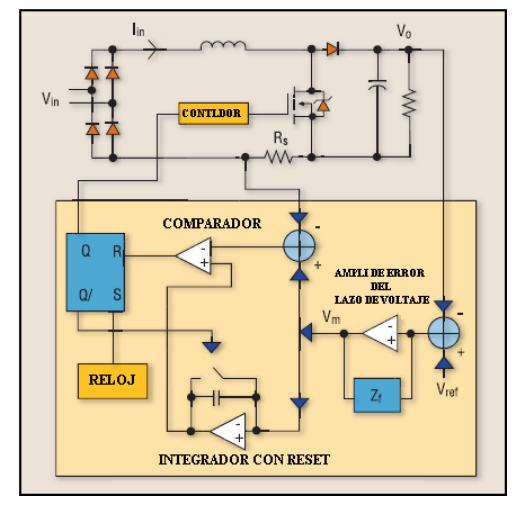

Fig. 3. One Cycle Control Technique [5].

A key aspect of the OCC control method is the fact that this ramp created by the integrator circuit is reset at the end of each switching cycle and the ramp starts again from zero at the beginning of the subsequent cycle. Accordingly, this method is aptly termed, OCC, or "One Cycle Control". The core of the One Cycle control is a resettable integrator. This block integrates the modulation voltage $V_{m}$ and is reset at the end of every switching cycle. Figures 4 and 5 shows the behavior of this block [6]-[9].

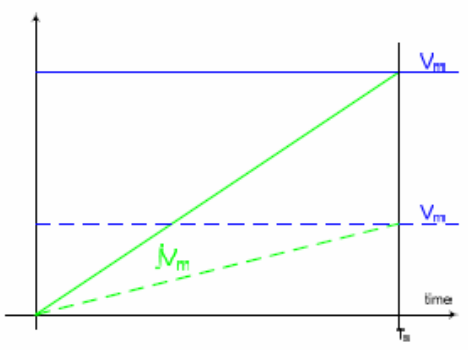

Fig. 4. Resettable integrator characteristic.

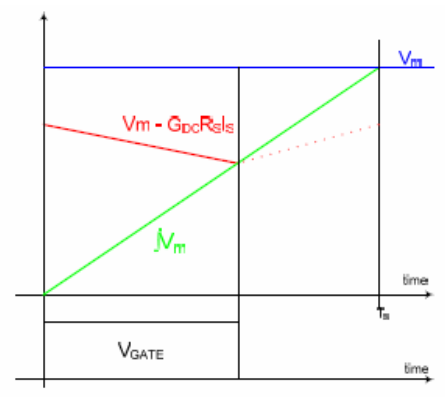

Fig. 5. PWM signal generation. 
An important characteristic is that integration time constant of the integrator must match the switching period, so that at the end of each cycle the ramp will match the integrated value.

In order to force the input current to have a sinusoidal or quasi - sinusoidal shape in the OCC, there is a need to have into account the following considerations:

The converter operates in the continuous conduction mode CCM, therefore according to equation (1):

$$
V_{\text {in }}=V_{o}(1-D)
$$

Due to the resettable integrator from figure 5:

$$
V_{m} x D=V m-I_{P K} x R_{s}
$$

Where $D$ is the duty cycle, $V_{m}$ is the modulation voltage, $I_{P K}$ is the instantaneous input peak current at the switching frequency and $R_{s}$ is the sense resistor. Reorganizing (3) gives

$$
I_{P K}=\frac{V_{m}-V_{m} x D}{R_{s}}
$$

Substituting (2) into (4) yields the resistor emulator.

$$
I_{P K}=\frac{V_{m} x V_{i n}}{V_{o} x R_{s}}
$$

Therefore, equation (5) demonstrates that the instantaneous $\mathrm{AC}$ line peak current is proportional to the input voltage, since $V_{m}, V_{O}$ and $R_{s}$ remain constant during each switching cycle. This means, that the Boost Converter behaves as a RE.

In this paper, the implementation of the control technique OCC for a Boost converter is made for the AC/DC conversion.

\section{DC/AC Conversion}

$\mathrm{DC} / \mathrm{AC}$ converters are known as inverters [10]. The main function of an inverter is to change an input DC voltage to an AC symmetric output voltage, with the desire amplitude and frequency. The inverter gain can be defined as the ratio between the $\mathrm{AC}$ output voltage and the input $\mathrm{DC}$ voltage. This type of conversion can be divided into two stages: the first one refers to the inverter topology and the second one refers to the modulation technique that will control the turn ON and OFF of the bridge switches.

Among the most common topologies for single-phase systems are the half bridge inverter, the full bridge inverter and the push-pull inverter. The configuration for the full bridge inverter is shown in figure 6. It is not possible to have two switches in the $\mathrm{ON}$ state simultaneously in the same leg,

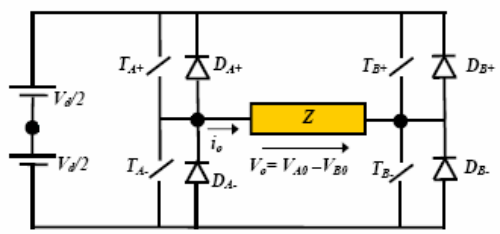

Fig. 6. Full Bridge Inverter.

This is the topology chosen as the configuration for the full bridge inverter of our DC/AC conversion, since in this topology, the fundamental component is twice the value of the half bridge inverters. There is a wide range of modulation techniques. For single-phase inverters the modulation techniques to use are: square wave modulation, sine wave modified modulation, also known as voltage cancellation modulation, and the carrier based sinusoidal pulse width modulation or CB-SPWM, for unipolar and bipolar case. The modulation technique chosen is the CB-SPWM with bipolar switching, due to its simplicity and easy implementation. So in order to get a sinusoid output voltage waveform to a desire frequency, a control sinusoid signal to this frequency is compared with a triangular wave. See figure 7 . The magnitudes relation between the reference and the triangular signal are defined as the modulation index, and it is given by:

$$
m_{a}=\frac{\hat{V}_{\text {Control }}}{\hat{V}_{T r i}}
$$

The frequency modulation ratio is defined as:

$$
m_{f}=\frac{f_{S}}{f_{1}}
$$

And it is specified greater than 21 in order to filtrate easily the output AC voltage harmonics.
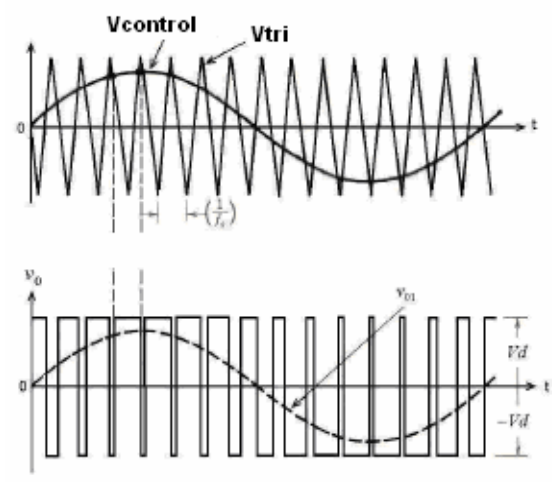

Fig. 7. PWM with Bipolar Switching.

The filter to eliminate the harmonics of the inverter output voltage is a low pass filter with LC configuration, as it is shown in figure 8 . 


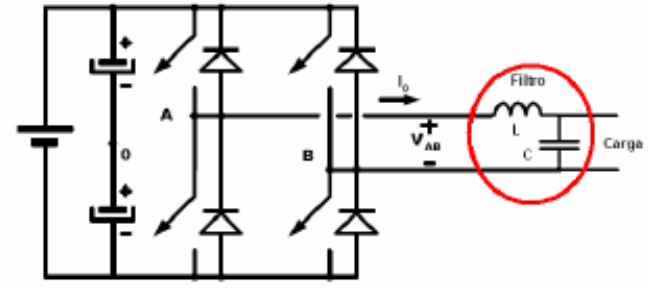

Fig. 8. LC filter.

The chosen values for the inductor and the capacitor for the filter are $\mathrm{L}=512 \mu \mathrm{H}$ and $\mathrm{C}=25 \mu \mathrm{F}$. The cutoff frequency of this filter is $1.4 \mathrm{KHz}$ and it is found with the following equation:

$$
f_{C}=\frac{1}{2 \pi \sqrt{L C}}
$$

\section{Specification and Implementation of the Double Single-Phase AC/DC/AC Converter with PFC}

\section{A. $A C / D C$ converter}

Figure 9 shows the schematic diagram of the Boost converter applying the OCC technique simulated in Orcad - Pspice.

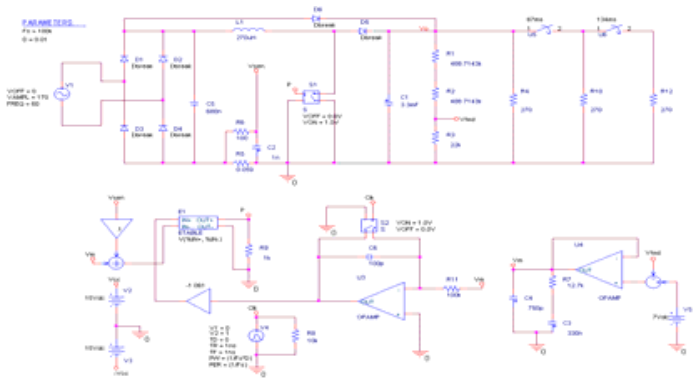

Fig. 9. Schematic diagram of the Boost Converter with the Control Technique OCC.

The input voltage (green trace) and the input current (red trace) are shown in figure 10, in which it is possible to demonstrate that the input current follows the input sinusoidal voltage proportionally, according to equation (5).

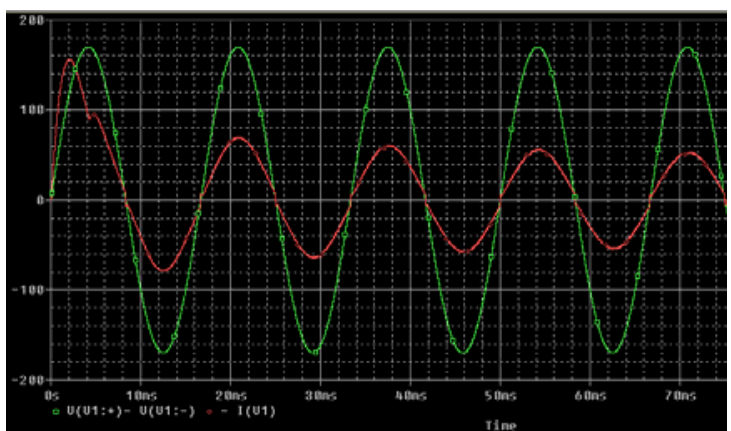

Fig. 10. Input current (red trace) and input voltage (green trace) for a full load (1200W).
The design of the Boost converter with PFC was developed with the new integrated circuit $\mu$ PFC IR1150S of International Rectifier. The circuit of the Boost converter applying the OCC technique and implementing an input EMI filter in order to reduce the electromagnetic noise caused by semiconductor devices is shown in figure 11. The Boost converter was implemented with the following specifications:

- Minimum input voltage: $100 \mathrm{~V}$

- Maximum input voltage: $120 \mathrm{~V}$

- Input frequency: $60 \mathrm{~Hz}$

- Switching frequency: $100 \mathrm{KHz}$

- Output power: $1200 \mathrm{~W}$

- Output voltage: $300 \mathrm{~V}$

- Minimum output voltage: $240 \mathrm{~V}$

- Efficiency: 97\%

- Start-up-time: $50 \mathrm{~ms}$

- Hold-up time: $30 \mathrm{~ms}$

The International Rectifier myPower Design Tool was used for both the control stage and the power stage design [11].

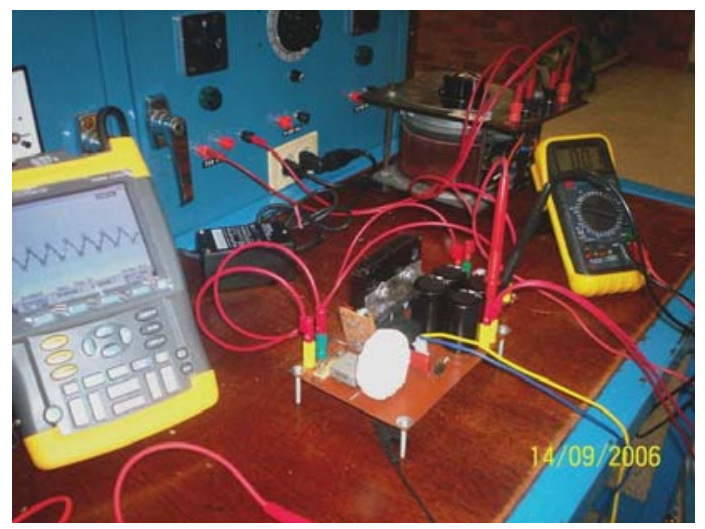

Fig. 11. AC/DC System conversion.

The input voltage and input current are shown in figure 12 , wherein it is possible to observe that they are in phase and that the system has a unity power factor to an output power of $250 \mathrm{~W}$.

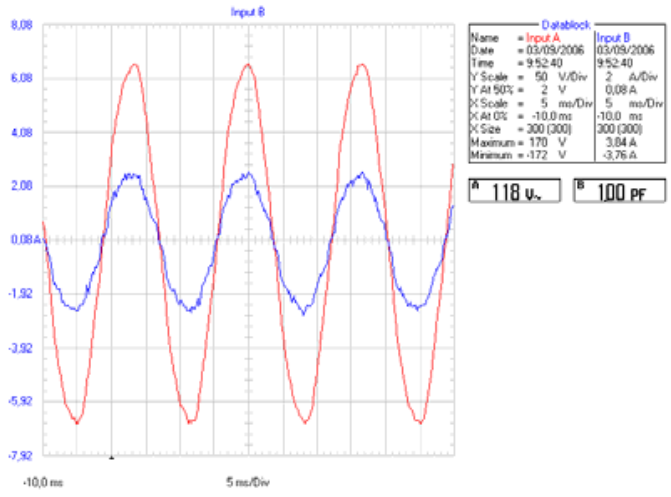

Fig. 12. Input voltage (red trace) and input current (blue trace). 
The harmonic spectrum of the input current as well as the total harmonic distortion (THD) of the system is shown in figure 13; there you can see that the THD is $6.21 \%$, which is really good.

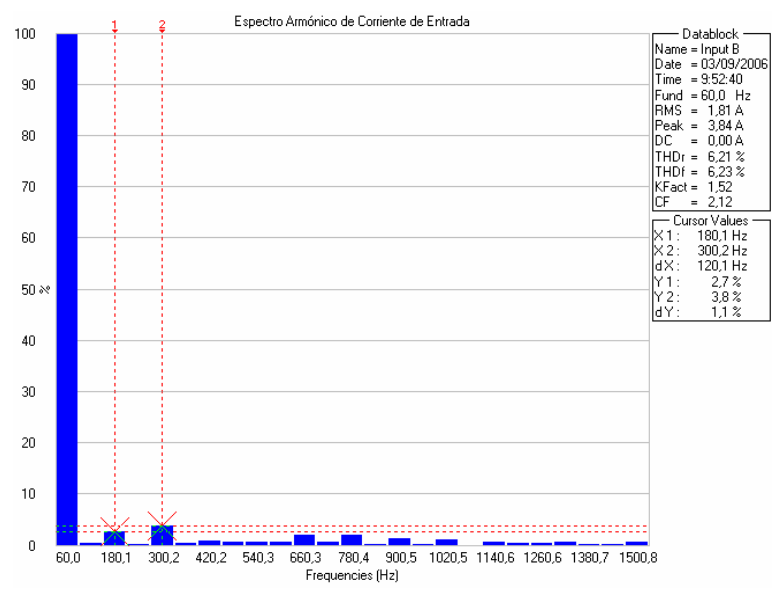

Fig. 13. Harmonic spectrum and total harmonic distortion (THD) of the input current.

Figure 14 and 15 show the transient response of the Boost converter, in which, the system was evaluated at different load conditions. There you can see the fast transient response with low voltage changing.

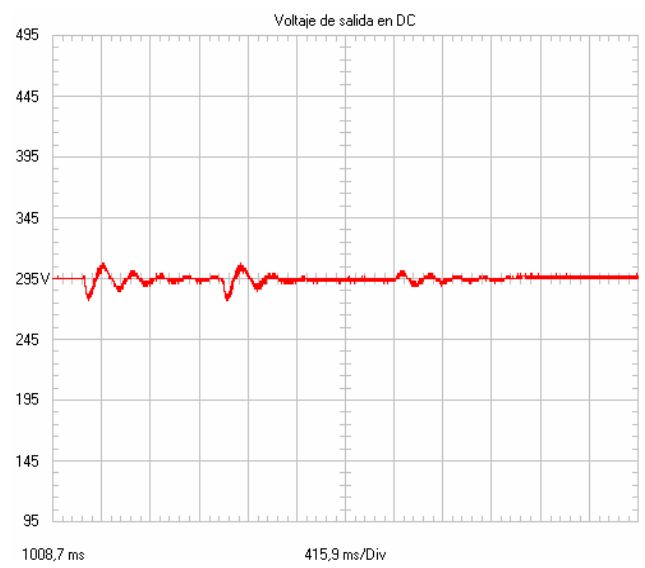

Fig. 14. Output DC Voltage.

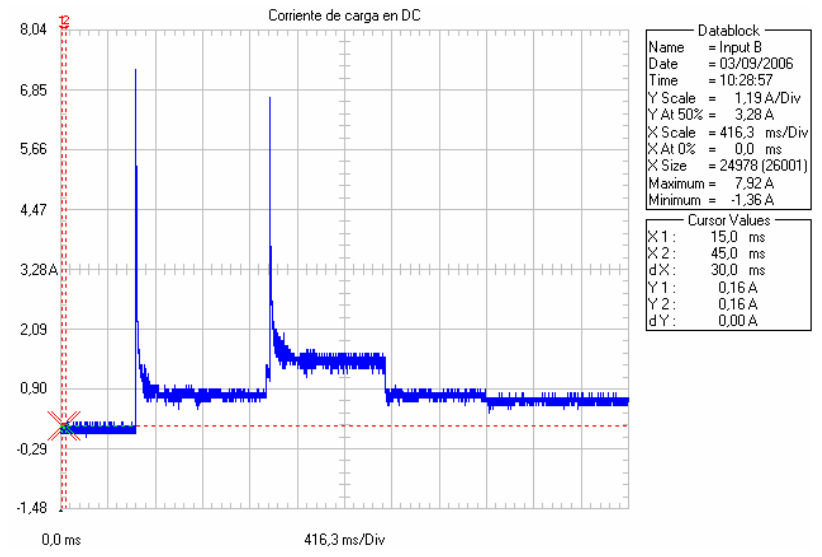

Fig. 15. Output DC Current.

\section{B. DC/AC converter}

A single-phase full bridge inverter was implemented for the power stage as it is shown in figure 6. Figure 16 shows the final power stage of the system.

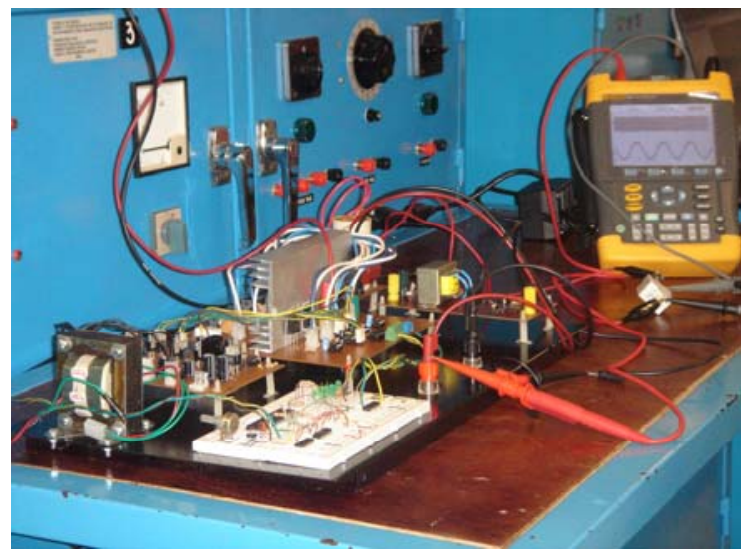

Fig. 16. Power stage of the full bridge inverter.

The output AC voltage and AC current (red trace and blue trace respectively) in open loop are shown in figure 17. The harmonic spectrum of the output voltage is shown in figure 18 , wherein it is possible to observe that the THD is $3.5 \%$, which is really good. The PWM signal (red trace) for the IGBT's and the ripple of the output voltage (blue trace) are shown in figure 19.

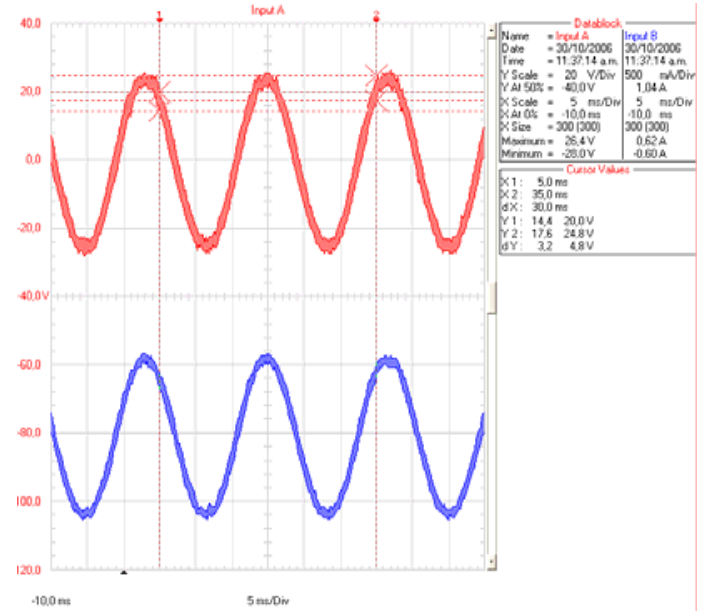

Fig. 17. Output AC Voltage and AC Current.

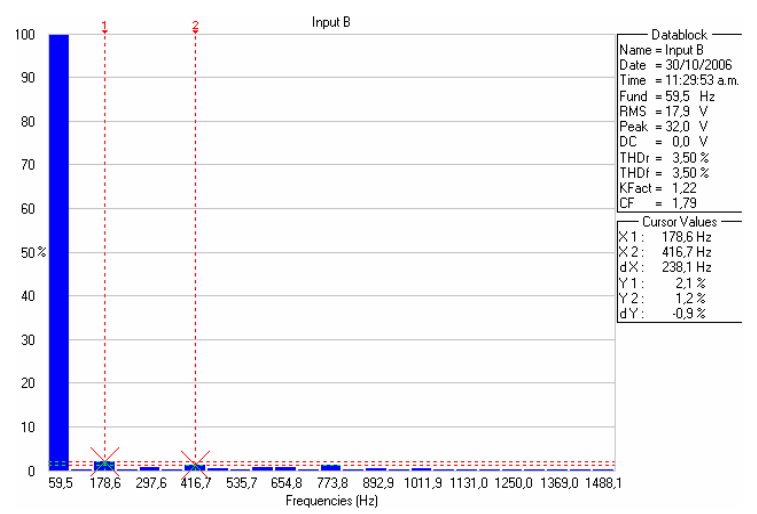

Fig. 18. Harmonic Spectrum of the Output AC Voltage. 


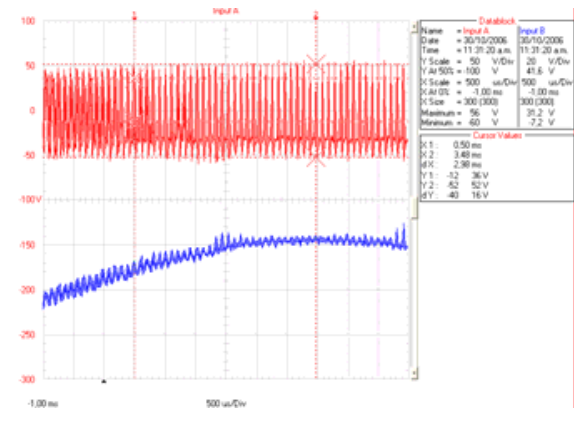

Fig. 19. PWM Signal for the IGBT's and Ripple of the

Output AC Voltage.

The control stage has been developed with the microcontroller PIC18F458 of Microchip, which was selected for this work due to its complete PWM module, which provides the necessary output in order to control the single-phase inverter. One way of generating the reference signal is implementing equation (9) in the microcontroller, which is not efficient. By such reason when it is desire to generate this signal using a microcontroller, the best way of doing it is implementing one table, which has discrete samples of the signal that is desired to be represented. In this paper, one table has been implemented, wherein the samples data correspond to equation (9).

$$
V_{\text {REF }}=127 * \sin (\theta)+128
$$

This table has a total of 82 data. The variation angle $\theta$ goes from $-90^{\circ}$ to $+90^{\circ}$. Sampling was made within this interval, since with these samples it is possible to generate the complete sinusoid signal. As the control strategy, the control with a single loop was chosen, with a SPWM control feedback of the type of natural sampling, wherein a detection of the output peak voltage is made. The controller design was made with the following parameters:

$$
\begin{aligned}
& \text { - } \mathrm{L}=512 \exp (-6) \\
& \text { - } \mathrm{C}=25 \exp (-6) \\
& \text { - } \mathrm{R}=10 \\
& \text { - } \mathrm{Vm}=300 \\
& \text { - } \mathrm{Vo}=170 \\
& \text { - } \mathrm{D}=0.5667
\end{aligned}
$$

Is very important to mention that the design was made with a resistive load. So the transfer function to simulate in MATLAB is:

$$
\frac{V o(s)}{D(s)}=\frac{\frac{V m}{L C}}{s^{2}+s \frac{1}{R C}+\frac{1}{L C}}
$$

The control has been designed in such a way that behaves as a first order system. We have added a real zero located in 222 , and in this case we have a gain of 5, which tell us that the transfer function of the PI controller is:

$$
5.003 \frac{(1+0.0045 s)}{s}
$$

In figure 20, the system close loop at full load can be observed, where we have added one pole in the origin and one zero near it.

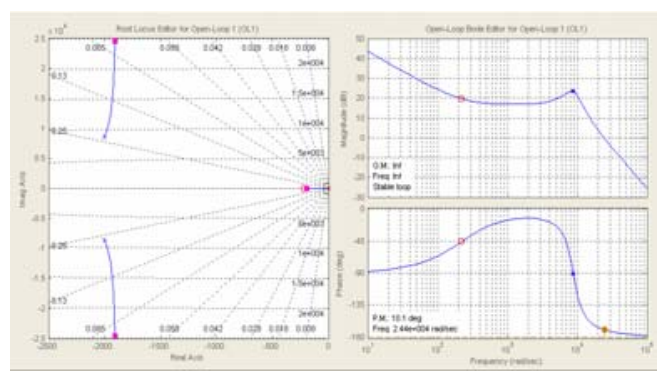

Fig.20. Close loop system at full load.

In the following figure the inverter with the PI close loop controller is shown, and it was simulated in Orcad Pspice.

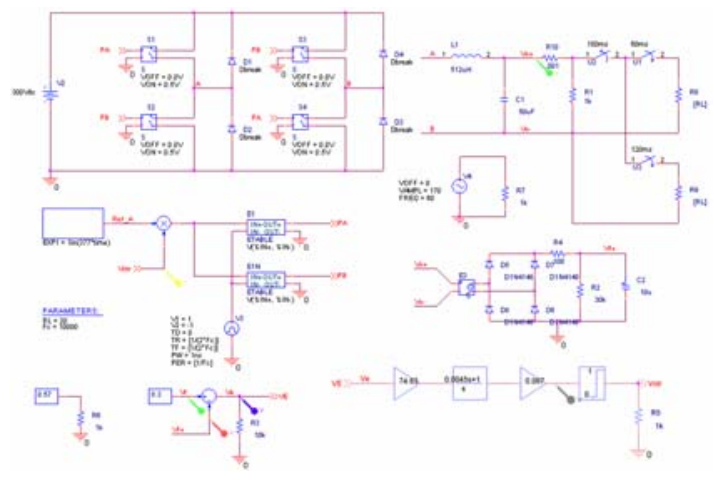

Fig. 21. Close loop Inverter.

Figure 22 shows the output AC current at different load conditions and figure 23 shows the behavior of the output $\mathrm{AC}$ voltage with the same system conditions, where you can see how the PI controller works in order to keep the output AC voltage constant.

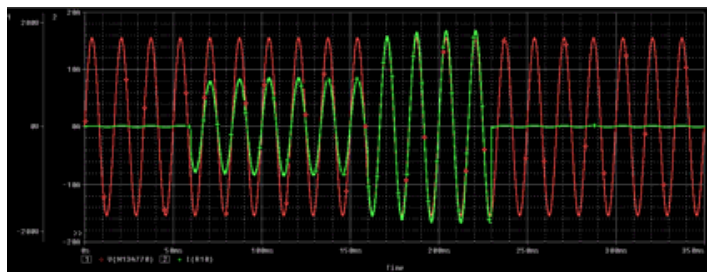

Fig. 22. Output AC current.

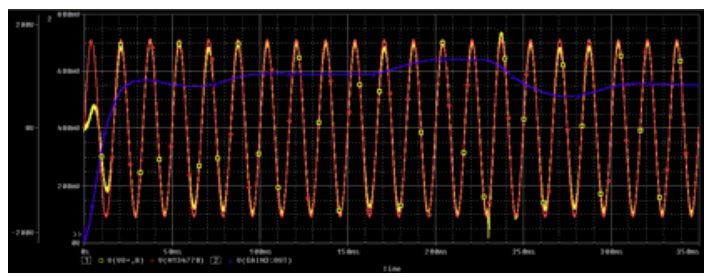

Fig. 23. Output AC voltage with close loop 


\section{Conclusion}

As a future work it is possible to develop an optimization of the PI controller implemented to the inverter system, with the purpose of increasing its output speed, since the natural sampling has a delay of approximately $8333 \mu \mathrm{s}$ in the sensed method. This can be improved using an instantaneous sampling of the signal, in other words, applying the predictive current control method.

The OCC control technique provides high power factor and low total harmonic distortion (THD) in order to conform to all requirements of harmonics generation over the AC grid.

Proper routing of critical circuit paths is elemental in optimum circuit performance and minimal system noise. Parasitic inductance resulting from long trace length in the power path can introduce noise spikes which can deteriorate performance to unacceptable levels. Thus, it is necessary to pay attention to the printed circuit board layout.

\section{Acknowledgment}

We would like to thank the Universidad Autonoma de Occidente and its Vicerectory of Investigation for allowing the development of the present work.

\section{References}

[1] Oscar Garcia, "Power Factor Correction in Single Phase Switching Converters", Universidad Politecnica de Madrid, España.

[2] Pavel Grasblum, "Implementing PFC Average Current Mode Control using the MC9512E128", Freescale Semiconductor, Application Note AN3052.

[3] Ron Brown, Marco Soldano, "One Cycle Control IC Simplifies PFC Designs", AC-DC Applications Group Internacional Rectifier Corp. $101 \mathrm{~N}$. Sepúlveda Blvd. El Segundo, CA, 90245 USA.

[4] K. Smedley and S. Cuk, "One Cycle Control of Switching Converters", IEEE Transactions on Power Electronics, Vol. 10, No. 6, pp 625-633, November 1995.

[5] David Morrison, "Controllers Build Compact PFC Circuits", Power Electronics Technology. April 2005

[6] R. Brown, M. Soldano, "PFC Converter with IR1150 One Cycle Control IC". Application Note AN-1077 International Rectifier.

[7] Z. Lai, Keyue Smedley. "A Family of Power Factor Correction Controllers". APEC '97. New York. IEEE 1997.

[8] K. Smedley. S. Cuk. "One Cycle Control of Switching Converters". 22nd Annual IEEE Power Electronics Specialists Conference (Cat. No.91CH3008-0), Cambridge, MA, 1991. pp. 88896. See also US patent 5,278,490 and IEEE Transactions on Power Electronics, Nov. 1995, Vol. 10, No. 6, P625-633.

[9] K. Smedley: "One-Cycle Controlled Switching Circuit” US Patent \# 5,278,490, Jan, 1994.
[10] N. Mohan, Power Electronics: Converters, Applications, and Design, john Wiley \& sons, Canada (1995), pp. 188-217.

[11] International Rectifier, "myPower Design Tool". Available: http://www.irf.com/design-center/mypower/ 